\title{
Remembering the Mathematics of Robert Brown Gardner
}

\author{
George R. Wilkens
}

\section{Mathematical Interests}

Robby Gardner, one of the organizers of this Summer Research Institute, passed away on May 5, 19981. Robby led an extremely active mathematical life producing more than fifty papers, three books, eighteen masters students and thirteen Ph.D students. He seemed to spend a sizeable fraction of every year traveling to conferences and meetings around the world. I was continually amazed by the number of mathematicians who had met Robby. He was one of those personalities that everyone, regardless of field, knew.

Robby was part of a long heritage of great differential geometers, tracing a direct line from Élie Cartan, through S.S. Chern, to himself. Robby's own mathematical interests clearly reflect this lineage. Even a casual observer of Robby's work could not help noticing the central role of exterior differential systems. Nor could anyone fail to notice Robby's enthusiasm for Cartan's work and his vigorous efforts to bring this work to a much larger audience. Robby certainly succeeded. Numerous "heirs to Cartan" dot the current mathematical landscape, and many of them have Robby as an ancestor on their family tree.

Robby's mathematical interests covered a number of areas. A list of these areas includes the following:

- geometric partial differential equations

- Cartan's equivalence method

- Cartan's method of moving frames

- exterior differential systems

I want to thank Robert Bryant, Niky Kamran and William Shadwick for their comments and support.

${ }^{1}$ To honor his contributions to graduate education, the UNC Mathematics Department created the Robert Brown Gardner Memorial Fund devoted to supporting graduate student activities. Contributions may be sent to Ms. Janet Farrell, Robert Brown Gardner Fund, Department of Mathematics, CB\#3250, University of North Carolina, Chapel Hill, NC 27599.

(C)1999 American Mathematical Society 
- submanifolds of Euclidean space

- integral geometry

- calculus of variations

- exterior algebra over commutative rings

- invariant theory

- control theory

- affine geometry

- Lie groups, Lie algebras and symmetries

- the geometry of shape in medical imaging.

I believe it is fair to say that Robby worked near the junction of geometry, algebra and analysis.

Before getting started, I want to issue a few apologies to the reader. Due to the limited time available to prepare this article, I will only be able to discuss some of the above topics. Further, time did not allow me to adequately refresh my memory of Robby's earlier work so my coverage will not be very balanced. Finally, I decided not to indicate which work is joint within the article, leaving that instead to the nearly complete bibliography. I hope this will not detract from the overall spirit of the article, and that the reader will forgive any shortcomings.

\section{Exterior Differential Systems}

Exterior differential systems (EDS) permeate nearly all of Robby's work. Many of his papers exemplify skillful and artistic applications of EDS. One of his very earliest papers Gar67 deals with differential systems in their own right. Specifically this paper discusses the invariants of one of the most commonly occurring differential systems, Pfaffian systems.

In this paper, Robby lays out tools he will use his entire life. The first invariant of a Pfaffian system is its Cauchy characteristics. This is an involutive distribution, and the Pfaffian system is locally generated by 1-forms involving only first integrals of the Cauchy system and their differentials. Moreover, a maximal independent set of first integrals of the Cauchy system gives the minimum number of variables required for any set of generators of the Pfaffian system. This number of variables, which is also the co-dimension of the Cauchy characteristic system, is called the class of the Pfaffian system.

In addition to the Cauchy characteristics, there is also the derived flag of a Pfaffian system. The length of the flag is called the derived length, and the co-dimensions of this flag of subspaces give further integer invariants, called the type numbers. Another basic integer invariant is the Engel rank. These integer invariants are not arbitrary, and Robby establishes a number of inequalities they must satisfy.

The real meat of the paper is contained in the last two sections. Robby shows how to intrinsically define a symmetric conformal tensor on Pfaffian systems with even co-dimension. In the case of co-dimension 4 , the conformal tensor is bilinear. The rank, index and any isotropic subspaces of this 
tensor are immediately seen to be invariants of the Pfaffian system. Robby applies these results to a single second order pde in the plane and shows that its classification as elliptic, parabolic, or hyperbolic corresponds exactly to the classification of the conformal tensor. Since this tensor is contact invariant, an immediate consequence is that the type of the pde is contact invariant. Robby goes on to show how the characteristic curves belonging to a solution of a hyperbolic or parabolic pde are completely determined by the isotropic subspaces of the conformal tensor.

In the early 1980s, Robby began participating in a new comprehensive treatment of the theory of exterior differential systems. This wonderful book $\left[\mathbf{B C G}^{+} \mathbf{9 1}\right]$ is now the standard reference for the subject. A tremendous amount of effort went into its production, and I remember a period of about ten years where it was regularly referenced in papers as "EDS, to appear." With ten chapters and nearly five hundred pages, the book covers a wide range of topics and strikes an appropriate balance between theory and application. Robby, of course, was a master at applying EDS and any reader wanting to supplement $\left[\mathbf{B C G}^{+} \mathbf{9 1}\right]$ with additional applications may consult the reference section of this article.

\section{Submanifolds and Integral Geometry}

Robby loved submanifold theory and integral geometry. He wrote a number of papers on these subjects including Gar69c, Gar70b, Gar70c, Gar71, Gar72b, Gar75b, Gar77b.

In Gar70b, Robby shows how the Dirichlet integral formula can be used to generate numerous integral formulas in differential geometry. In addition to showing how this technique leads to several established results, Robby proves a new rigidity theorem for Euclidean spheres.

Robby goes to town in Gar77b] by digging into the study of arbitrary co-dimension submanifolds of Euclidean space. As one might guess from the title, New viewpoints in the geometry of submanifolds of $\mathbf{R}^{N}$, the new viewpoints are Robby's own and they are wonderfully original. Robby develops the basic invariants in a way that is both geometrically and algebraically natural. He defines new invariants, gives new meaning to old ones, and develops new methods for studying submanifolds.

\section{Invariant Theory}

While this section is very short, it would be very remiss not to mention one of Robby's most scholarly works Gar80. Interestingly, it is a review of a book on invariant theory. The book is only reviewed in the last paragraph while the rest of the article is a review of invariant theory itself.

Robby brings the reader through a historical development of the subject in a mere ten pages. He traces this development from the naive beginnings with Cayley and Sylvester to the post-Hilbert period of then present 1980. While, at first glance, this attention to invariant theory might seem out of 
place in the work of a differential geometer, Robby understood its central importance in the early development of differential geometry and, indeed, how important the interplay between the two subjects was. However, Robby's interest was not just scholarly. He certainly viewed invariant theory as the ultimate technique in tensor analysis and used it wherever possible.

\section{Geometric PDE2}

Robby's papers contain beautiful developments of EDS concepts applied to the geometric study of PDEs. In Gar69a and Gar69b, Robby defined and studied a rather broad notion of a Cauchy problem for Pfaffian systems. He was notably able to prove an existence theorem for the Cauchy problem in the $C^{\infty}$ category for Pfaffian systems admitting what Robby called $k$-stable vector fields. These are vector fields which essentially stabilize the Pfaffian system after $k$ iterations of the Lie derivative. They can thus be thought of as natural generalizations of Cauchy characteristics. He then defined a powerful general notion of characteristic and focal systems for Pfaffian systems endowed with distinguished invariant subsystems, such as the maximal isotropic subsystems associated to an intrinsic conformal symmetric bilinear form. The fundamental examples are the Monge and Friedrichs characteristics for the Pfaffian systems associated to scalar second-order pdes or first-order determined systems in the plane. This enabled him to prove in Gar69b and Gar77a several elegant existence theorems and finite escape-time theorems for the smooth Cauchy problem for non-linear parabolic equations. Paper Gar79] gives a very elegant explanation in EDS terms of a notoriously difficult memoir of Goursat on Bäcklund and Laplace transformations for scalar second-order hyperbolic pdes in the plane. Papers GK93, GK95 and GK97 contain further applications of this body of ideas to second-order hyperbolic pdes and first-order determined systems in two independent variables.

\section{Control Theory}

Robby began his work in control theory in the early 1980s. He had a long interest in this vital area of twentieth-century research, especially in the work by the very active group in geometric control. Problems related to the classification of orbits of control systems with respect to various transformation groups held a special interest for Robby. He was, after all, one of the few experts at that time in a technique ideally suited to study such problems.

In a series of four talks given at a conference on geometric control theory at Michigan Tech, Robby introduced exterior differential systems and Cartan's equivalence method to a new audience. The corresponding paper Gar83 showcases Robby's original perspectives on differential geometry, and I think it is an important mile marker in his career. On reexamining

\footnotetext{
${ }^{2}$ I am extremely grateful to Niky Kamran for contributing this section.
} 
this paper, I can not help but feel the connection between his early papers (e.g. Gar67, Gar68, Gar69b) and his later books on the method of equivalence Gar89 and on exterior differential systems $\mathbf{B C G}^{+} \mathbf{9 1}$.

Robby began to study the orbits of control systems under the action of the pseudo-group of (static) feedback transformations. One of the principal tools of this investigation was Cartan's equivalence method. A series of papers describing normal forms, new integration methods, geometric isomorphisms, relationships with classical geometries, and a universal bundle for control systems issued forth.

Much of the early work is summarized very nicely in the paper [Gar91. This paper begins by describing the representation of the feedback equivalence problem as a $G$-structure equivalence problem. Following that is a discussion of the $G$-structure equivalence associated to constrained variational problems of the sort found in Phillip Griffiths' book on calculus of variations. One immediately observes that these $G$-structures are formally identical which suggests a geometric isomorphism between the two problems. Some low dimensional examples are examined in detail. In addition to identifying systems with control-linear and linear normal forms, systems with special geometric structures are also identified. Examples include systems with intrinsically attached pseudo-Riemannian metrics and contact structures.

Robby generated tremendous interest in his applications of the equivalence method to control theory. Interest was so great that in 1988 Robby was invited to be the principal speaker at a joint AMS-SIAM CBMS regional conference at Texas Tech. Robby gave a series of ten lectures on Cartan's method of equivalence. Robby covered the general theory and presented numerous applications to differential geometry and control theory. Robby's book [Gar89] was the first to clearly exhibit the algorithmic nature of the equivalence method. His book remains the best one-stop source for general theory, examples and computational technique.

A particularly important piece of work emanated from studying the feedback orbits of Brunovsky normal forms. While the problem of identifying which non-linear systems are feedback equivalent to a Brunovsky normal form was already solved, some interesting new results were obtained. For instance in GSW89a it was shown that a Brunovsky normal form is characterized by its symmetry subgroup. Following this was a series of two papers GS91a], GS92] showing how the symmetries give rise to a new algorithm for finding a particular feedback transformation to linearize the original non-linear system. This algorithm, called the GS-algorithm, was a substantial improvement over then existing methods. The GS-algorithm identifies a set of Frobenius systems, minimal in dimension and number, to be integrated. A maximal independent set of solutions to these systems forms part of a linearizing coordinate system. The remaining coordinates are then determined by routine differentiation and linear algebra. The efficiency 
of the GS-algorithm led to its adoption by NASA as part of an automatic flight controller.

A beautiful normal form theorem lies at the heart of GS-algorithm. The spirit of this new theorem is very similar to other normal form theorems in exterior differential systems, such as the Engel, Goursat and Bryant normal forms (see $\mathbf{G a r 8 3}$ or $\mathbf{B C G}^{+} \mathbf{9 1}$ for details on these normal forms.) I believe that this result inspired some of the work by Shankar Sastry, Richard Murray and their colleagues on applications of exterior differential systems.

The paper on control structures [BG95] provided an intrinsic and global representation of control systems in terms of an affine co-distribution in the co-tangent space of a manifold. The co-distribution must satisfy some reasonable necessary conditions. The interesting properties of these structures are that they naturally determine a manifold of states, their presentation in local coordinates has the expected form, and the given co-distribution is realized as the pull-back of a canonical co-distribution on the punctured tangent bundle to the state manifold. This last property represents the universal bundle construction belonging to control structures. One of the main technical advantages of control structures is that tools like the method of equivalence can be directly and easily applied.

The work on control structures developed from the interpretation of a control system as a subbundle of the tangent bundle to state space, and the interpretation of a feedback transformation as the canonical extension of a state space diffeomorphism to the tangent bundle. From these interpretations, Robby observed that a control system embeds a submanifold in each tangent space and that a feedback transformation induces a linear action on each of these submanifolds. He reasoned that an important first step in understanding the geometry of the subbundle is to understand the geometry in each fiber. The next step is to piece the fiber geometries together in a way that exhibits their evolution over state space.

The fiber geometry can be described as the study of submanifolds of a finite dimensional vector space under the action of the full general linear group. This turns out to be a subject in classical differential geometry called centro-affine geometry. While this geometry is classical, far more attention had been given to the study of affine geometry and equi-affine geometry. Theorems in centro-affine geometry were relatively scarce. Additionally, the existing theorems had to be adapted to their intended applications to the feedback equivalence problem. With that in mind, Robby established the basic theorems for curves and hyper-surfaces in centro-affine geometry [GW97.

Some applications of centro-affine geometry are found in GW93 and GW96b. These papers revisit the geometric isomorphism between control systems and variational problems. They show that the feedback invariants for a co-dimension 1 control system are completely determined by the centroaffine geometry of the fibers. The evolution of the fiber geometry across 
the state space is uniquely determined. The relationship between these codimension 1 systems and Finsler geometry becomes clear. This connection shows how to transport the same analysis to Finsler structures. In this case the centro-affine fiber geometry determines Chern's connection for Finsler geometry. This geometric isomorphism with Finsler geometry also explains the appearance of pseudo-Riemannian metrics which were observed earlier.

The applications just cited use the centro-affine structure of hypersurfaces to study co-dimension 1 systems. The centro-affine structure of curves was being applied to the study of scalar control systems. That work is unfinished, but I intend to continue that project and report on this part of Robby's work.

\section{Farewell to a Friend}

My goal in this article has been to give the reader a sample of the substantial contributions Robby made to mathematics. I also want to take this opportunity to say something about Robby as a person. Many of us knew Robby through his passions: his love for his family, his loves of fine food and fine wine, his love of music, his love of travel, his ardent stamp collecting hobby, and his commitment to excellence in all things.

At the center of all these passions was a deeply caring person. This is what I think of first when I think of my friend. Robby's office door was always open, and this simple symbol reflects so much of Robby himself. I never saw him turn anyone away, regardless of how busy he was. Robby would guide the inexperienced, nurture the talented, and clear obstructions wherever he could. He was a marvelous teacher, mentor, colleague and friend. He touched the lives of so many people and in ways too numerous to list. Robby leaves a legacy of humanity as well as one of mathematics, and with very fond memories I bid him adieu.

\section{References}

$\left[\mathrm{BCG}^{+} 91\right]$ R. L. Bryant, S. S. Chern, R. B. Gardner, H. L. Goldschmidt, and P. A. Griffiths, Exterior differential systems, Mathematical Sciences Research Institute Publications, vol. 18, Springer-Verlag, New York, 1991.

[BG95] Robert L. Bryant and Robert B. Gardner, Control structures, Geometry in nonlinear control and differential inclusions (Warsaw, 1993), Banach Center Publ., vol. 32, Polish Acad. Sci., Warsaw, 1995, pp. 111-121.

$\left[\mathrm{EGM}^{+} 94\right]$ D. Eberly, R. Gardner, B. Morse, S. Pizer, and C. Scharlach, Ridges for image analysis, J. Math. Imaging and Vision 4 (1994), 353-373.

[Gar65] Robert B. Gardner, Differential geometric methods in partial differential equations, Ph.D. thesis, University of California at Berkeley, 1965.

[Gar67] Robert B. Gardner, Invariants of Pfaffian systems, Trans. Amer. Math. Soc. 126 (1967), 514-533.

[Gar68] Robert B. Gardner, Some applications of the retraction theorem in exterior algebra, J. Differential Geometry 2 (1968), 25-31.

[Gar69a] Robert B. Gardner, The Cauchy problem for Pfaffian systems, Comm. Pure Appl. Math. 22 (1969), 587-596. 
[Gar69b] Robert B. Gardner, A differential geometric generalization of characteristics, Comm. Pure Appl. Math. 22 (1969), 597-626.

[Gar69c] Robert B. Gardner, An integral formula for immersions in euclidean space, J. Differential Geometry 3 (1969), 245-252.

[Gar70a] Robert B. Gardner, A differential geometric approach to characteristics, Global Analysis (Proc. Sympos. Pure Math., Vol. XVI, Berkeley, Calif., 1968), Amer. Math. Soc., Providence, R.I., 1970, pp. 179-186.

[Gar70b] Robert B. Gardner, The Dirichlet integral in differential geometry, Global Analysis (Proc. Sympos. Pure Math., Vol. XV, Berkeley, Calif., 1968), Amer. Math. Soc., Providence, R.I., 1970, pp. 231-237.

[Gar70c] Robert B. Gardner, The geometry of subscalar pairs of metrics, Proc. Carolina Conf. on Holomorphic Mappings and Minimal Surfaces (Chapel Hill, N.C., 1970), Dept. of Math., Univ. of North Carolina, Chapel Hill, N.C., 1970, pp. 29-42.

[Gar71] Robert B. Gardner, The technique of integral formulas in the geometry of immersions, Differentialgeometrie im Grossen (Tagung, Math. Forschungsinst., Oberwolfach, 1969), Bibliographisches Inst., Mannheim, 1971, pp. 127-144. Ber. Math. Forschungsinst. Oberwolfach, No. 4.

[Gar72a] Robert B. Gardner, Lectures on exterior algebras over commutative rings, Department of Mathematics, University of North Carolina, Chapel Hill, N. C., 1972 .

[Gar72b] Robert B. Gardner, Subscalar pairs of metrics and hypersurfaces with a nondegenerate second fundamental form, J. Differential Geometry 6 (1972), 437458, Collection of articles dedicated to S. S. Chern and D. C. Spencer on their sixtieth birthdays.

[Gar74] R. B. Gardner, The influence of Carathéodory on differential systems, Proceedings of the C. Carathéodory International Symposium (Athens, 1973), Greek Math. Soc., Athens, 1974, pp. 146-153.

[Gar75a] Robert B. Gardner, The fundamental theorem of vector relative invariants, J. Algebra 36 (1975), no. 2, 314-318.

[Gar75b] Robert B. Gardner, Geometry of submanifolds of euclidean spaces, Differential geometry (Proc. Sympos. Pure Math., Vol. XXVII, Stanford Univ., Stanford, Calif., 1973), Part 1, Amer. Math. Soc., Providence, R.I., 1975, pp. 125-134.

[Gar77a] Robert B. Gardner, Differential geometric viewpoints on the development of shock waves, The 1976 Ames Research Center (NASA) Conference on the Geometric Theory of Non-linear Waves (Moffett Field, Calif., 1976), Math. Sci. Press, Brookline, Mass., 1977, pp. 89-104. Lie Groups: History, Frontiers and Appl., Vol. VI.

[Gar77b] Robert B. Gardner, New viewpoints in the geometry of submanifolds of $\mathbf{R}^{N}$, Bull. Amer. Math. Soc. 83 (1977), no. 1, 1-35.

[Gar78] Robert B. Gardner, Differential geometry and foliations: the Godbillon-Vey invariant and the Bott-Pasternack vanishing-theorems, Differential topology, foliations and Gelfand-Fuks cohomology (Proc. Sympos., Pontifícia Univ. Católica, Rio de Janeiro, 1976), Springer, Berlin, 1978, pp. 75-94. Lecture Notes in Math., Vol. 652.

[Gar79] Robert B. Gardner, Constructing Bäcklund transformations, Partial differential equations and geometry (Proc. Conf., Park City, Utah, 1977), Lecture Notes in Pure and Appl. Math., vol. 48, Dekker, New York, 1979, pp. 77-89.

[Gar80] Robert B. Gardner, Invariant theory, Bull. Amer. Math. Soc. 12 (1980), 246256.

[Gar83] Robert B. Gardner, Differential geometric methods interfacing control theory, Differential geometric control theory (Houghton, Mich., 1982), Progr. Math., vol. 27, Birkhäuser, Boston, Mass., 1983, pp. 117-180. 
[Gar85] Robert B. Gardner, Differential equations and the method of equivalence, Proc. IEEE Conference on Decision and Control, December 1985, Fort Lauderdale, FL, pp. 1777-1778.

[Gar88] Robert B. Gardner, The principal components construction applied to affine unimodular repère mobile, Resultate Math. 13 (1988), no. 3-4, 235-239, Reprinted in Affine Differentialgeometrie [235-239, Tech. Univ. Berlin, Berlin, 1988].

[Gar89] Robert B. Gardner, The method of equivalence and its applications, CBMSNSF Regional Conference Series in Applied Mathematics, vol. 58, Society for Industrial and Applied Mathematics (SIAM), Philadelphia, PA, 1989.

[Gar91] Robert B. Gardner, The geometry of nonlinear control systems, Differential geometry, Pitman Monographs Surveys Pure Appl. Math., vol. 52, Longman Sci. Tech., Harlow, 1991, pp. 179-198.

[Gar76] Robert B. Gardner, Modules admitting determinants, Linear and Multilinear Algebra 3 (1975/76), no. 3, 209-214.

[GK93] R. B. Gardner and N. Kamran, Characteristics and the geometry of hyperbolic equations in the plane, J. Differential Equations 104 (1993), no. 1, 60-116.

[GK95] R. B. Gardner and N. Kamran, Normal forms and focal systems for determined systems of two first-order partial differential equations in the plane, Indiana Univ. Math. J. 44 (1995), no. 4, 1127-1162.

[GK97] R. B. Gardner and N. Kamran, Exterior differential systems and normal forms of parabolic systems, Advances in mathematical sciences: CRM's 25 years (Montreal, PQ, 1994), CRM Proc. Lecture Notes, vol. 11, Amer. Math. Soc., Providence, RI, 1997, pp. 215-230.

[GKS95] Robert B. Gardner, Marcus Kriele, and Udo Simon, Generalized spherical functions on projectively flat manifolds, Results Math. 27 (1995), no. 1-2, 4150, Festschrift dedicated to Katsumi Nomizu on his 70th birthday (Leuven, 1994; Brussels, 1994).

[GS85] Robert B. Gardner and William F. Shadwick, Equivalence of one-dimensional Lagrangian field theories in the plane. I, Global differential geometry and global analysis 1984 (Berlin, 1984), Lecture Notes in Math., vol. 1156, Springer, Berlin-New York, 1985, pp. 154-179.

[GS87a] R. B. Gardner and W. F. Shadwick, Feedback equivalence of control systems, Systems Control Lett. 8 (1987), no. 5, 463-465.

[GS87b] R. B. Gardner and W. F. Shadwick, Overdetermined equivalence problems with an application to feedback equivalence, Differential geometry: the interface between pure and applied mathematics (San Antonio, Tex., 1986), Contemp. Math., vol. 68, Amer. Math. Soc., Providence, RI, 1987, pp. 111-119.

[GS87c] R. B. Gardner and W. F. Shadwick, A simple characterization of the contact system on $J^{k}(E)$, Rocky Mountain J. Math. 17 (1987), no. 1, 19-21.

[GS90a] R. B. Gardner and W. F. Shadwick, Feedback equivalence for general control systems, Systems Control Lett. 15 (1990), no. 1, 15-23.

[GS90b] R. B. Gardner and W. F. Shadwick, Symmetry and the implementation of feedback linearization, Systems Control Lett. 15 (1990), no. 1, 25-33.

[GS91a] Robert B. Gardner and William F. Shadwick, An algorithm for feedback linearization, Differential Geom. Appl. 1 (1991), no. 2, 153-158.

[GS91b] Robert B. Gardner and William F. Shadwick, An equivalence problem for a two-form and a vector field on $\mathbf{R}^{3}$, Differential geometry, global analysis, and topology (Halifax, NS, 1990), CMS Conf. Proc., vol. 12, Amer. Math. Soc., Providence, RI, 1991, pp. 41-50.

[GS92] Robert B. Gardner and William F. Shadwick, The GS algorithm for exact linearization to Brunovsky normal form, IEEE Trans. Automat. Control 37 (1992), no. 2, 224-230. 
[GSW89a] R. B. Gardner, W. F. Shadwick, and G. R. Wilkens, Feedback equivalence and symmetries of Brunowski normal forms, Dynamics and control of multibody systems (Brunswick, ME, 1988), Contemp. Math., vol. 97, Amer. Math. Soc., Providence, RI, 1989, pp. 115-130.

[GSW89b] Robert B. Gardner, William F. Shadwick, and George R. Wilkens, A geometric isomorphism with applications to closed loop controls, SIAM J. Control Optim. 27 (1989), no. 6, 1361-1368.

[GW93] Robert B. Gardner and George R. Wilkens, Classical geometries arising in feedback equivalence, Proc. IEEE Conference on Decision and Control, December 1993, San Antonio, TX, pp. 3437-3440.

[GW96a] Robert B. Gardner and George R. Wilkens, Preface for "Applications of Finsler geometry to control theory", Finsler geometry (Seattle, WA, 1995), Contemp. Math., vol. 196, Amer. Math. Soc., Providence, RI, 1996, pp. 227229.

[GW96b] Robert B. Gardner and George R. Wilkens, A pseudo-group isomorphism between control systems and certain generalized Finsler structures, Finsler geometry (Seattle, WA, 1995), Contemp. Math., vol. 196, Amer. Math. Soc., Providence, RI, 1996, pp. 231-243.

[GW97] Robert B. Gardner and George R. Wilkens, The fundamental theorems of curves and hypersurfaces in centro-affine geometry, Bull. Belg. Math. Soc. Simon Stevin 4 (1997), no. 3, 379-401.

[OPGP91] W. R. Oliver, S. M. Pizer, R. B. Gardner, and K. B. Pryzwansky, Evaluation of cytologic deformation by boundary-driven energy minimization, Prog. Clin. Biol. Res. 363 (1991), 355-368.

Department of Mathematics, University of Hawait, 2565 The Mall, HonOLULU, HI 96822-2273

E-mail address: grw@math.hawaii.edu 